\title{
Comparison of Working Length Determination in Teeth with Vital Pulps with Digital Radiographs and Four Electronic Apex Locators. An In Vivo Study
}

\author{
Jorge Paredes Vieyra ${ }^{1 *}$, Fernando Calleja Casillas ${ }^{2}$ and Acosta Guardado Julieta ${ }^{3}$ \\ ${ }^{1}$ San Ysidro California, USA \\ ${ }_{2}^{2}$ Department of Celular Biology, Universidad Autonoma de Baja California, Mexico \\ ${ }^{3}$ Endodontist, Universidad Autónoma de Baja California, México
}

*Corresponding author: Jorge Paredes Vieyra, 710E San Ysidro Blvd. suite "A" \#1513, San Ysidro California 92173, USA.

Received Date: November 29, 2018

Published Date: December 12, 2018

\begin{abstract}
Introduction: The aim of this study was to evaluate in vivo the accuracy and predictability of four EALs for determining working length as compared to radiographs: RootZX, Apex ID, Joypex 5 and Propex Pixi.

Methods: One hundred and twenty patients (247 canals) contributed in the study. The measurements obtained by the four EALs and radiographs relative to the actual location of the AC were compared using a paired samples t test, $\mathrm{X}^{2}$ test.

Results: For anterior teeth, EALs and radiographs located the minor foramen $87 \%, 75 \%, 75 \%, 87 \%$ and $62 \%$ of the time, respectively. For premolar teeth, EALs and radiographs located the minor foramen $92.85 \%, 85.71 \%, 78.57 \%, 85.71 \%$ and $57.14 \%$ of the time, respectively. For molar teeth, the Root ZX, EALs and radiographs located the minor foramen 90\%, 85\%, 80\%, 85\% and $70 \%$ of the time, respectively. There was no statistically significant difference between the four EALs but there was a difference bet the EALs and radiographs. $\mathrm{p}=0.05$.

Conclusion: Under clinical conditions the EALs identified the apical constriction (minor foramen) with high degree of accuracy. EAL were more accurate, compared to radiographs with the potential to greatly reduce the risk of instrumenting and filling beyond the apical foramen.
\end{abstract}

Keywords: Apical foramen, Constriction, Root apex, Root ZX, Mini apex, Joypex

\section{Introduction}

The success of root canal treatment has to be done on the correct implementation of all stages of the procedure from diagnosis to the canal filling. Among these steps, the determination of working length (WL) is a challenge and depends on the anatomy of the root end. Filling root canals should not extend beyond the tooth apex. The apical constriction (AC), is a reasonable site for working length since it frequently corresponds with the constricted width of the canal [1,2]. Dummer PHM, et al. [3] recognized that it is hard to locate the $\mathrm{AC}$ in vivo with reliance because of its location.

The cementodentinal junction has been recommended as the histological site for working length because it represents the shift among two tissues [4]. The situation of this point is commonly recognized as being $0.50 \mathrm{~mm}$ to $0.75 \mathrm{~mm}$ cervical to the apical foramen [5] but, as with the $\mathrm{AC}$, the precise position of the cementodentinal junction is hard to find in vivo. Frequently, this point is considered to be co-located with the minor foramen [6]; nonetheless, this is not all the time the case [3].

WL is termed as "the distance from a coronal reference point to the point at which canal preparation and filling should terminate" [2]. The radiographic position of WL has restrictions that complicate its understanding and conception. Even when a radiographic technique is recommended modifications of images has been found to be around 5\% [7].

Variations in determine the WL finish in over or under instrumentation due to the variations in distance among the apical foramen and the radiographic apex [8]. 
The use of electronic apex locators (EALs) to determine the WL has become common [9]. Periapical radiographs have been considered as the traditional and most appropriate way of gaining suggestion on the configuration of the root canal and its adjacent tissues [10-13]. Digital radiography still has limitations including image distortion, superposition of roots and adjacent anatomical structures, and higher radiation exposure compared to electronic methods [9].

Periapical radiographs are still an aid for diagnosis, for working films (e.g. calculating the working length of root canals, fitting gutta percha cones), to confirm the final fill, and for followup comparisons at recall examinations. The mechanism of EALs based on the principle of frequency dependent impedance, EALs have been trusted with the basic role in determining the WL Though, even operating the same principle, modern EALs operate mechanisms that vary from the initially planned ones.

Several use the impedance quotient principle such as Root ZX (0.4 and $8 \mathrm{kHz}$ ) (J. Morita, Tokyo, Japan) [14,15]and Apex ID (0.5 and $5 \mathrm{kHz}$ ) (SybronEndo, Orange, CA) [16,17]. Joypex 5, Denjoy Dental Corporation, Changsha City, China and Propex Pixi (Dentsply Sirona, Maillefer Instruments Holding Sàrl Chemin du Verger 3 CH-1338 Ballaigues Switzerland) [18,19] use the square root of impedances in 2 frequencies.

EALs have the potential to enable the identification of the device inside the canal, allowing more precise in vivo determination of working length (WL) [20]. Recent EALs have a great consistency and high accuracy and in finding the major AF regardless of the internal environment [21].

EALs have the ability to reduce the number of radiographs used through root canal therapy [22]. Modern EALs can locate the AF and the AC with extraordinary precision. The accuracy of measurement might also differ on the file size and the anatomy of the root canal and apical foramen.

Considering the value of the precise determination of WL in the success of endodontic therapy [23], the aim of this study is to evaluate in vivo the accuracy and predictability of four modern and precise EALs: Root ZX, Apex ID, Joypex 5 and Propex Pixi for determining WL as compared to digital radiographs. The null hypothesis considered was that no significant differences would be found between the EALs.

\section{Materials and Methods}

G*Power version 3.0.10 (Heinrich Heine, Universität Düsseldorf, Düsseldorf, Germany) were used to calculate sample size by using the Wilcoxon-Mann-Whitney test [17]. A total of 42 samples were indicated as the best size required for observing important changes.

This clinical study was achieved at the Autonomous University of Baja California, School of Dentistry, Tijuana, Mexico. The study protocol was accepted by the Ethics Committee (71/2018) and conducted in agreement with the ethical principles of the last update of the Declaration of Helsinki [24]. All participants were informed on the goals and strategy of the study and written informed consent permissions were gained previously treatment.
The patients' inclusion parameters were the absence of radiographic signs of apical periodontitis with a diagnosis of irreversible pulpitis established by pulp sensitivity testing with heat and cold. The corresponding author performed thermal pulp examination, and the radiographic diagnosis was established by 2 certified endodontists. This study includes only vital teeth in order to avoid false response of the periapical zone due to a periapical pathology or inflammation of the surrounding tissues.

Further clinical requirements for patients' inclusion were: 1) the purposes and necessities of the study were understood and spontaneously accepted. 2) Sufficient coronal structure for rubber dam isolation. 3) No previous root canal treatment.

Exclusion parameters were previously root canal treated teeth, gravidity, impossibility to obtain patient's approval, patients who didn't complete inclusion criteria, patients younger than 18 years. Non-vital teeth and teeth with apical pathosis, endodontic retreatment, root resorption, undeveloped apex, or a root canal with severe curvature $\left(>35^{\circ}\right)$ or a radiographically untraceable canal path to the minor foramen were all rejected from this research.

The diagnosis of vital pulp was confirmed by the presence of bleeding after gaining access to the pulp chamber. If the thermal test was positive and there was bleeding following pulp exposure, the tooth was established as vital.

\section{Patient selection}

Forty-two of 54 patients ( 31 women and 11 men) aged 18-55 years were incorporated in this study while 15 were excluded as not meeting the inclusion criteria. The 42 teeth (116 canals) allocated to each protocol were adequate to confirm an essential sample size. All teeth responded positively to thermal exams and were extracted for periodontal or prosthodontic purposes.

All clinical procedures and measurements were performed by a single experienced operator. After local anesthesia, rubber dam isolation and access cavity (\#331 carbide bur, Dentsply Maillefer USA) and refined with an Endo Z bur (Dentsply Maillefer). The root canals were flared cervically with \#1-\#2 Orifice Shapers (Dentsply Maillefer USA) using $2.5 \% \mathrm{NaOCl}$ for irrigation.

Manual K Flex-R files (Moyco, Union Broach) size \#10 and \#15 were used for the negotiation of the canals, after which the cervical and middle thirds were prepared with ProTaper SX, S1 and S2 instruments (Dentsply Maillefer) inserted apically up to $5.0 \mathrm{~mm}$ short of the apparent canal length. After preflaring (establishment of a canal path to the minor foramen), the apical constriction was standardized with K Flex-R file size \#20 using $2.5 \% \mathrm{NaOCl}$ as an irrigating solution. The apical constriction of each tooth was identified first with the four EALs and then radiographically.

\section{Treatment protocol}

All the EALs electronically and radiographically (Schick Technologies, NY, USA) measured the total sample $(\mathrm{N}=42)$. The EALs were used with a full load. A single qualified endodontist who previously calibrated the EALs performed all the electronic WLs with manual $\mathrm{K}$ files calibrated to the required apical limit. Measurements were considered to be valid if the reading remained stable for at least 5 seconds. The order of use of the EALs followed 
an alternate sequence to permit all of the equipment to be used the same number of periods as the first one.

For the Root ZX. The AC was situated with this EAL according to the company's procedure guidelines [14]. A size \#15 K-file was advanced in the canal until the LCD displayed a flashing bar between APEX and 0.5 with corresponding symbol and a flashing tooth with the audible signal indicating that the AC had been located. Two silicone stoppers (to prevent file movement) on the file were placed at the reference point. The instrument was removed from the canal and the length measured to the nearest $0.01 \mathrm{~mm}$ with a digital caliper (Mitutoyo America Corporation Aurora IL). This was the insertion length.

For the Apex ID. The AC was detected with the Apex ID per the manufacturer's Instruction Guidelines (C2018 Kerr Corporation, USA). The same size \#15 file used for the Root ZX. The Apex ID displays this position graphically and numerically (" 0.0 "), then the insertion length was measured as above. For the Joypex 5 until the device reach the " 0.0 " level, according to the manufacturer's recommendations. Then the insertion length was measured as above.

For the Propex Pixi. The Propex Pixi has a LED that represents the AC position (0.0), then the insertion length was measured as described in the previous protocol.

After completing all the readings with the EALs, the instruments had their rubber stops adjusted to the occlusal references of the teeth. The teeth were extracted and the roots were analyzed with an operating microscope for the presence of fractures or any other type of change that would exclude the tooth.

After the four EAL had established the AC on the same tooth with the same size \#15 file the AC was positioned radiographically by advancing the file until its tip was assumed to be $1.0 \mathrm{~mm}$ from the radiographic apex as estimated from the initial radiograph. A digital radiograph was exposed and if the tip was not $1.0 \mathrm{~mm}$ from

Table 1: Distribution of 42 teeth (116 canals). the radiographic apex the file was repositioned, and an additional radiograph was taken to ensure that it was. The file was removed and after the insertion length was measured it was re-inserted to this length (1 $\mathrm{mm}$ from the radiographic apex) and cemented in place with Fuji II LC dual-cure glass ionomer cement (GC Corp, Tokyo, Japan). The file handle was removed with a high-speed bur and after the tooth was extracted without disturbing the file, it was placed in $5.25 \% \mathrm{NaOCl}$ for $20 \mathrm{~min}$ to clean the root surface and stored in a $1 \%$ Thymol solution.

The tooth was removed from the thymol and with the file in place, the apical $5 \mathrm{~mm}$ of the root was ground parallel to the long axis of the canal with a fine diamond bur and abrasive discs. When the file became visible, additional dentine was eliminated while viewing the process under 30X magnification with an OPMI Pico microscope (Carl Zeiss, Munich, Germany) until the instrument tip and the apical AF were in focus. A digital photograph was taken and stored in Adobe Photoshop cc 2017 (Adobe Systems Inc., San Jose, CA, USA).

The distance of the instrument tip from the AC (narrowest part of the canal) was measured and documented as being $-1.0 \mathrm{~mm}$ or $-0.5 \mathrm{~mm}$ from the $\mathrm{AC}$; at the $\mathrm{AC}$, or $+0.5 \mathrm{~mm}$ from the $\mathrm{AC}$. A negative symbol (-) indicated a file short of the AC; A positive symbol (+) indicated it was long of the AC. Since the insertion length was already known, the actual length to the $\mathrm{AC}$ was determined by adding or subtracting the distance of the file tip from the $\mathrm{AC}$ to the insertion length.

After the actual length (distance from the reference point to the $\mathrm{AC}$ ) was established the distance of the instrument tip from the AC was calculated for the four EAL by comparing the insertion length with the actual length. The difference was recorded as -1.0 or $-0.5 \mathrm{~mm}$, etc., from the AC as shown in Tables 1-4. The distances of the instrument tip from the AC obtained by the 4 EALs and the distances obtained radiographically were compared using a paired samples $\mathrm{t}$-test and a repeated measure ANOVA evaluation at the 0.05 level of significance.

\begin{tabular}{|c|c|c|c|c|}
\hline \multirow{2}{*}{} & \multicolumn{2}{|c|}{ Tooth } & \multicolumn{2}{c|}{ No. of Canals } \\
\cline { 2 - 5 } & Maxillary & Mandibular & Maxillary & 0 \\
\hline Central Incisor & 4 & 0 & 4 & 0 \\
\hline Lateral Incisor & 3 & 0 & 1 & 0 \\
\hline Canine & 1 & 0 & 8 & 4 \\
\hline $1^{\text {st }}$ Premolar & 4 & 2 & 10 & 6 \\
\hline $2^{\text {nd }}$ Premolar & 5 & 3 & 20 & 28 \\
\hline $1^{\text {st }}$ Molar & 5 & 7 & 16 & 16 \\
\hline $2^{\text {nd }}$ Molar & 4 & 4 & 62 & 54 \\
\hline Total & 26 & 16 & & \\
\hline
\end{tabular}

Table 2: Distance of file tip from the apical constriction determined by Root ZX, Apex ID, Joypex 5, Propex Pixi and Radiograph (8 anterior teeth: 8 canals).

\begin{tabular}{|c|c|c|c|c|c|}
\hline Group & $\begin{array}{c}1.0 \mathrm{~m} \mathrm{n}=8(\%) \\
\text { Variance Std. Deviation }\end{array}$ & $\begin{array}{c}-0.5 \mathrm{~mm} \mathrm{n}=8(\%) \\
\text { Variance Std. Deviation }\end{array}$ & $\begin{array}{c}\text { AC } n=8(\%) \text { Variance } \\
\text { Std. Deviation }\end{array}$ & $\begin{array}{c}+0.5 \mathrm{~mm} \mathrm{n}=8(\%) \\
\text { Variance Std. Deviation }\end{array}$ & $\begin{array}{c}+1.0 \mathrm{~mm} \mathrm{n}=8(\%) \\
\text { Variance Std. Deviation }\end{array}$ \\
\hline \multirow[t]{2}{*}{ Root ZX } & - & - & $7(87.5 \%)$ & $1(12.5 \%)$ & - \\
\hline & & & $0.099 / 0.314$ & $0.100 / 0.340$ & \\
\hline \multirow[t]{2}{*}{ Apex ID } & - & - & $6(75 \%)$ & $2(25 \%)$ & - \\
\hline & & & $0.139 / 0.373$ & $0.151 / 0.380$ & \\
\hline
\end{tabular}




\begin{tabular}{|c|c|c|c|c|c|}
\hline Joypex 5 & - & - & $6(75 \%)$ & $2(25 \%)$ & - \\
\hline & & & $0.139 / 0.373$ & $0.151 / 0.380$ & \\
\hline Propex Pixi & - & - & $7(87.5 \%)$ & $1(12.5 \%)$ & - \\
\hline & - & - & $0.099 / 0.314$ & $0.100 / 0.340$ & \\
\hline Radiograph & & & $5(62.5 \%)$ & $1(12.5 \%)$ & $2(25 \%)$ \\
\hline & & & $0.115 / 0.373$ & $0.810 / 0.94$ & $0.153 / 0.382$ \\
\hline
\end{tabular}

AC: Apical Constriction (+) and (-) values indicate file tip beyond (+) or short (-) of the AC. $p=0.05$

Table 3: Distance of file tip from the apical constriction determined by Root ZX, Apex ID, Joypex 5, Propex Pixi and Radiograph (14 premolars: 28 canals).

\begin{tabular}{|c|c|c|c|c|c|}
\hline Distance from AC (mm) & Root ZX n = 14 (\%) & Apex ID $\mathbf{n}=\mathbf{1 4}(\mathbf{\%})$ & Joypex 5 n= 14 (\%) & Propex Pixi n= 14 (\%) & Radiograph \\
\hline-1.0 & - & - & - & - & - \\
\hline-0.5 & - & - & - & $12(85.71 \%)$ & - \\
\hline AC & $13(92.85 \%)$ & $12(85.71 \%)$ & $11(78.57 \%)$ & $2(14.28 \%)$ & $5(35.71 \%)$ \\
\hline+0.5 & $1(7.14 \%)$ & $2(14.28 \%)$ & $3(21.42 \%)$ & & $1(7.14 \%)$ \\
\hline+1.0 & & & & & \\
\hline
\end{tabular}

AC: Apical Constriction (+) and (-) values indicate file tip beyond (+) or short (-) of the AC. $p=0.05$

Table 4: Distance of file tip from the apical constriction determined by Root ZX, Apex ID, Joypex 5, Propex Pixi and Radiograph (20 molars: 80 canals).

\begin{tabular}{|c|c|c|c|c|c|}
\hline Distance from AC $(\mathbf{m m})$ & Root ZX $\mathbf{n = 2 0}$ & Apex ID $\mathbf{n = 2 0}$ & Joypex 5 n=20 & Propex Pixi $\mathbf{n = 2 0}$ & Radiograph $\mathbf{n = 2 0}$ \\
\hline-1.0 & - & - & - & - & - \\
\hline-0.5 & - & - & - & - & - \\
\hline AC & $18(90 \%)$ & $17(85 \%)$ & $16(80 \%)$ & $17(85 \%)$ & $14(70 \%)$ \\
\hline 0.5 & $2(10 \%)$ & $3(15 \%)$ & $4(20 \%)$ & $3(15 \%)$ & $5(25 \%)$ \\
\hline 1 & & & & & $1(5 \%)$ \\
\hline
\end{tabular}

AC: Apical Constriction (+) and (-) values indicate file tip beyond (+) or short (-) of the AC. $p=0.05$

\section{Results}

The percentage of measurements at the $\mathrm{AC} ; 0.5 \mathrm{~mm}$ and $1.0 \mathrm{~mm}$ short of the AC; $0.5 \mathrm{~mm}$ and $1.0 \mathrm{~mm}$ through the $\mathrm{AC}$ was recorded as shown in Tables 2-4.

For anteriors, premolars, and molars: no measurements were $1.0 \mathrm{~mm}$ short of the Apical constriction. For anteriors and premolars: No measurements were $0.5 \mathrm{~mm}$ short of the Apical constriction (Tables 2-4). In addition, none of the EAL measurements were $1.0 \mathrm{~mm}$ through the AC whereas with digital radiographs it was $25 \%$ in anterior teeth, $7.14 \%$ in bicuspids and $5 \%$ in molars respectively. A WL $1.0 \mathrm{~mm}$ through the AC will, in some cases, result in instrumenting and filling beyond the foramen. A WL $0.5 \mathrm{~mm}$ short of, or at the radiographic apex, would further increase the likelihood of this happening. For anterior teeth, EALs and radiographs located the minor foramen $87 \%, 75 \%, 75 \%, 87 \%$ and $62 \%$ of the time, respectively. For premolar teeth, EALs and radiographs located the minor foramen $92.85 \%, 85.71 \%, 78.57 \%$, $85.71 \%$ and $57.14 \%$ of the time, respectively. For molar teeth, the Root ZX, EALs and radiographs located the minor foramen $90 \%$, $85 \%, 80 \%, 85 \%$ and $70 \%$ of the time, respectively. There was no statistically significant difference among the four EALS ( $p=0.05)$ but it was statically significant between them and digital radiographs.

\section{Discussion}

The goal of this study was to evaluate in vivo the accuracy and predictability of four EALs: Root ZX, Apex ID, Joypex 5 and Propex Pixi for determining WL as compared to digital radiographs. Usually, radiographs have been the primary means for determining WL.
However, they have inherent restrictions, being two-dimensional images of three-D objects.

Both methods were used to investigate conventional radiographs, digital images and EALs solely or in comparison to each other. However, neither in vitro nor in vivo results can be a true representative of clinical situations in which the whole treatment is done in the mouth [14]. On the other hand, randomized clinical studies may provide high-level of evidence for clinical practice since they reflect a truly clinical condition.

All measurements were conducted by the same experienced and trained operator, thus eliminating the possibility of operator bias. A WL is gained radiographically by setting the tip of an instrument a certain distance, commonly $1.0 \mathrm{~mm}$, from the radiographic apex. This method lacks accuracy because the $1.0 \mathrm{~mm}$ is measured from the end of the root (radiographic apex) rather than the apical foramen. Wrbas KT, et al. [25] urged caution to avoid overestimating WL because the AF frequently was not at the apex. Gutiérrez \& Aguayo [8] recorded a wide variability in distances between the foramen and radiographic apex ranging from 0.20 to $3.40 \mathrm{~mm}$.

There is a common agreement in the endodontic community that WL should be situated at the AC. Kuttler Y [1], found that the apical constriction averaged 0.5 to $0.75 \mathrm{~mm}$ from the AF and that the distance increased with age because of cementum deposition. Chapman C [26] and Dummer PMH, et al. [3] found that the AC was located $0.5-1.0 \mathrm{~mm}$ from the apex in $92 \%$ and $95 \%$ of the inspected teeth, separately. Hassanien EE, et al. [27] detected the apical 
constriction an average distance of $1.2 \mathrm{~mm}$ from the AF. In light of these studies, it would seem that there is a plenty justification to establish a WL $1.0 \mathrm{~mm}$ short of the radiographic apex.

Unfortunately, this statement is not always accurate in locating the apical constriction and care should be used because a WL 1 mm short of the radiographic apex and supposed to be close to the apical constriction may actually be beyond the AF. When this occurs, an instrument passing through a necrotic pulp and through the foramen will most likely carry microorganisms and its products into the apical area $[28,29]$. Receiving an indication from an EAL when the apical constriction is located would be very beneficial in preventing this mishap.

The use of an EAL to calculate WL has extended acceptance. Even though the user must be alert of the possible sources of miscalculation (metallic restorations, salivary contamination, dehydration, etc.), the present study and other scientific papers have presented that the precision of EALs is better than radiographs [7,30-32]. In general, our study also agrees with others [33] that EALs are more accurate than radiographs and greatly reduce the chance of instrumenting and filling short of or beyond the apical foramen.

Being $1.0 \mathrm{~mm}$ through the apical constriction increases the risk of over instrumentation and filling. In this study, using a radiographically determined WL $1.0 \mathrm{~mm}$ from the radiographic apex resulted in $19.4 \%$ of the anterior teeth, $43.75 \%$ of the premolars, and $35.13 \%$ of the molars being $1.0 \mathrm{~mm}$ through the AC. In comparison, no EALs measurements for anterior, premolar and molar teeth were $1.0 \mathrm{~mm}$ through the apical constriction. Thus, the null hypothesis was accepted.

The limitations and disadvantages of using only the radiographic method to determine WL are well known. Superimposition of anatomic structures could contribute to increasing a clinician's inaccuracy in locating WL. Additionally, prior to digital radiography, radiation was an even greater concern. However, since the joint use of radiographs together with EAL results in greater accuracy [34], radiographic verification of WL length is still desirable $[35,36]$.

So, it can be said that the accurate determination of WL will be dependent on the ability of the clinician to read radiographs, an excellent monitor to see and interpret digital radiographs, correct assumption of apical constriction with the help of an EAL, handling and using a combination of all methods, application of logic, knowledge of anatomy of the canals and especially the apical third and tactile sense.

\section{Conclusion}

Thus, based on the results of this study it can be concluded that EALs identified the AC with greater accuracy and predictability than digital radiographs. All achieved a clinically acceptable determination of WL and were significantly more accurate than radiographs.

\section{Acknowledgment}

None.

\section{Conflict of Interest}

The author deny any conflicts of interest related to this study.

\section{References}

1. Kuttler Y (1955) Microscopic investigation of root apexes. J Am Dent Assoc 50(5): 544-552.

2. (2003) American Association of Endodontists. Glossary of Endodontic Terms $7^{\text {th }}$ edn

3. Dummer PMH, McGinn JH, Rees DG (1984) The position and topography of the apical canal constriction and apical foramen. Int Endod J 17(4): 192-198.

4. Grove CJ (1931) The value of the dentinocemental junction in pulp canal surgery. J Dent Res 11: 466-468.

5. Ricucci D, Langeland K (1988) Apical limit of root canal instrumentation and obturation, part 2. A histological study. Int Endod J 31(6): 394-409.

6. Stein TJ, Corcoran JF, Zillich RM (1990) The influence of the major and minor foramen diameters on apical electronic probe measurements. J Endod 16(11): 520-522.

7. Van de Voorde HE, Bjorndahl AM (1969) Estimating endodontic "working length" with paralleling radiographs. Oral Surg Oral Pathol Oral Med 27(1): 106-110.

8. Gutiérrez JH, Aguayo P (1995) Apical foraminal openings in Human teeth. Number and location. Oral Surg Oral Med Oral Pathol Oral Radiol Endodon 79(6): 769-777.

9. Heo MS, Han DH, An BM, Huh KH, Yi WJ, et al. (2008) Effect of ambient light and bit depth of digital radiograph on observer performance in determination of endodontic file positioning. Oral Surg Oral Med Oral Pathol Oral Radiol Endodon 105(2): 239-244.

10. Bramante CM, Berbert A (1974) A critical evaluation of some methods of determining tooth length. Oral Surg Oral Med Oral Pathol 37(3): 463473.

11. Custer LE (1918) Exact method of locating the apical foramen. J Nat Dent Assoc 5(8): 815-819.

12. Suzuki K (1942) Experimental study on iontophoresis. J Japan Estomat 16: 411-429.

13. Nekoofar MN, Ghandi MM, Hayes SJ, Dummer PM (2006) The fundamental operating principles of electronic root canal length measurement devices. Int Endod J 39(8): 595-609.

14. Root ZX II (2005) Operation Instructions. J Morita Corp, Kyoto, Japan.

15. Altunbas, Kuştarcı A, Toyoğlu M (2017) The influence of various irrigants on the accuracy of 2 electronic apex locators in locating simulated root perforations. J Endod 43(3): 439-442.

16. Sybron Endo (2006) Elements Diagnostic: Instruction Guidelines. Glendora, CA: 1-3

17. Vasconcelos BC, Bueno MM, Luna-Cruz SM, Duarte MA, Fernandes CA et al. (2013) Accuracy of five electronic foramen locators with different operating systems: an ex vivo study. J Appl Oral Sci 21(2): 132-137.

18. Marigo L, Gervasi GL, Somma F, Squeo G, Castagnola R, et al. (2016) Comparison of two electronic apex locators on human cadavers. Clin Oral Investig 20(7): 1547-1550.

19. Ustun Y, Aslan T, Sekerci AE, Sagsen B (2016) Evaluation of the reliability of cone-beam computed tomography scanning and electronic apex locator measurements in working length determination of teeth with large periapical lesions. J Endod 42(9): 1334-1337.

20. Gordon MP, Chandler NP (2004) Electronic apex locators: a review. Int Endod J 37(7): 425-437.

21. Jenkins JA, Walker WA, Schindler WG, Flores CM (2001) An in vitro evaluation of the accuracy of the Root ZX in the presence of various irrigants. J Endod 27(3): 209-211.

22. Pagavino G, Pace R, Baccetti T (1998) An SEM study of in vivo accuracy of the Root ZX electronic apex locator. J Endod 24(6): 438-441.

23. Cianconi L, Angotti V, Felici R, Conte G, Mancini M (2010) Accuracy of three electronic apex locators compared with digital radiography: an ex vivo study. J Endod 36(12): 2003-2007. 
24. (2000) World Medical Association. Declaration of Helsinki: ethical principles for medical research involving human subjects. JADA 284(243): 3043-3045.

25. Wrbas KT, Ziegler AA, Altenburger M, Schirrmeister JF (2007) In vivo comparison of working length determination with two electronic apex locators. Int Endod J 40(2): 133-138.

26. Chapman C (1969) A microscopic study of the apical region of human anterior teeth. J Brit Endod Soc 3: 52-55

27. Hassanien EE, Hashem A, Chalfin H (2008) Histomorphometric study of the root apex of mandibular premolar teeth: an attempt to correlate working length measured with electronic and radiograph methods to various anatomic positions in the apical portion of the canal. J Endod 34(4): 408-412.

28. Siqueira JF Jr, Rŏças IN, Favieri A, Machado AG, Gahyva SM, et al. (2002) Incidence of postoperative pain after intracanal procedures based on an antimicrobial strategy. J Endod 28(6): 457-460.

29. Siqueira JF, Barnett F (2004) Interappointment pain: mechanisms, diagnosis, and treatment. Endod Top 7(1): 93-109.

30. Pratten DH, Mc Donald NJ (1996) Comparison of radiographic and electronic working lengths. J Endod 22(4): 173-176.
31. Venturi M, Breschi L (2007) A comparison between two electronic apex locators: an ex vivo investigation. Int Endod J 40(5): 362-373.

32. Shabahang S, Goon WWY, Gluskin AH (1996) An in vivo evaluation of Root ZX electronic apex locator. J Endod 22(11): 616-618.

33. Usun 0, Topuz O, Tinaz C, Nekoofar MH, Dummer PM (2008) Accuracy of two root canal length measurement devices integrated into rotary endodontic motors when removing gutta-percha from root-filled teeth. Int Endod J 41(9): 725-732.

34. El Ayouti A, Weiger R, Lost C (2002) The ability of Root ZX apex locator to reduce the frequency of overestimated radiographic working length. J Endod 28(2): 116-119.

35. Fouad AF, Krell KV, Mc Kendry DJ, Koorbusch GF, Olson RA (1990) Clinical evaluation of five electronic root canal length measuring instruments. J Endod 16(9): 446-449.

36. Vieyra JP, Acosta J, Mondaca JM (2010) Comparison of working length determination with radiographs and two electronic apex locators. Int Endod J 43(1): 16-20. 